\title{
ESTRATÉGIA AVANÇADA DE SINTONIA PARA CONTROLE POR MATRIZ DINÂMICA COM APLICAÇÃO NÃO-LINEAR
}

\author{
Daniel Cavalcanti Jeronymo*, Antonio Augusto Rodrigues Coelho* \\ * Universidade Federal de Santa Catarina \\ Pós-Graduação em Engenharia de Automação e Sistemas \\ Campus Universitário Reitor João David Ferreira Lima, C.P. 476, CEP 88040-900 \\ Florianópolis, SC, Brasil
}

Emails: dcavalcanti@bigfoot.com, aarc@das.ufsc.br

\begin{abstract}
In this paper an autotuning method for Dynamic Matrix Control (DMC) is presented with application to single-input single-output (SISO) processes which can be approximated by a first-order-plus-dead-time model. In order to validate the method a non-linear control valve system described by a Wiener process is simulated. This process was identified by a Hammerstein model so DMC could perform non-linear control. The proposed tuning method has its performance compared to another method commonly found in literature, showing more conservative results regarding smoothness of the control action while maintaining adequate performance on settling time.
\end{abstract}

Keywords - dynamic matrix control, non linear control, first-order-plus-dead-time, autotuning.

Resumo - Neste trabalho é apresentado um método de sintonia automática (autotuning) para o DMC com aplicação em processos SISO que possam ser aproximados por um modelo FOPDT. Para validação do método é simulado um sistema não-linear de válvula de controle descrito por um processo Wiener. Um processo Hammerstein é identificado a partir deste sistema para efetuar o controle não-linear pelo DMC. O método de sintonia proposto tem seu desempenho comparado com outro método popularmente encontrado na literatura, demonstrando resultados mais conservativos quanto a suavidade da ação de controle enquanto mantem bom desempenho relativo ao tempo de acomodação.

Palavras-chave— controle por matriz dinâmica, controle não linear, FOPDT, sintonia automática.

\section{Introdução}

O algoritmo do controle preditivo por matriz dinâmica (Dynamic Matrix Control - DMC) tem se mantido essencialmente o mesmo desde a sua concepção nos trabalhos originais por (C.R. and P.S., 2001; Cutler et al., 1982). As inovações nesta área do controle preditivo baseado em modelo são apresentadas fundamentalmente para dois problemas diferentes: i) a representação matemática do modelo, e ii) a sintonia de parâmetros, os horizontes e fator de supressão de movimento, do controlador.

A sintonia de parâmetros do DMC é problemática já que não há maneira analítica exata para obtenção dos parâmetros que produzam a melhor resposta desejada. Em (Qin and Badgwell, 2003) é apresentada uma extensa revisão do DMC com aplicações industriais e em (Kokate et al., 2010) uma revisão da literatura quanto a métodos de sintonia do DMC para processos SISO. Dentre os métodos de sintonia automática (autotuning) se destaca o de Shridhar and Cooper (1997) por sua facilidade de uso a partir de um modelo firstorder-plus-dead-time (FOPDT).

Neste trabalho é apresentado um método de sintonia para o DMC considerando plantas que possam ser aproximadas por um modelo FOPDT. Inicialmente, parte-se de considerações de uma realização mínima do DMC para que seja possível efetuar uma análise do comportamento do sistema em malha fechada. Em seguida a sintonia é expandida para melhor contemplar o modelo do processo, utilizando o tempo de resposta e atraso do tempo morto. Observa-se que esta sintonia expandida é um híbrido da sintonia do fator de supressão de movimentos desta proposta com o método de sintonia de horizontes de Shridhar and Cooper (1997).

O restante deste trabalho está organizado da seguinte maneira. A seção 2 faz uma breve introdução ao DMC e seus parâmetros. A seção 3 apresenta os fundamentos do método de sintonia proposto, assim como um método de sintonia manual e um método de sintonia automática (autotuning). A seção 4 apresenta um estudo de caso de uma planta não-linear de válvula de controle. A seção 5 apresenta os resultados obtidos pelo método proposto e os compara com o método de Shridhar and Cooper (1997). Finalmente, a seção 6 apresenta as considerações finais sobre o trabalho e trabalhos futuros.

\section{Controlador por Matriz Dinâmica}

O controle preditivo por matriz dinâmica (C.R. and P.S., 2001; Cutler et al., 1982) foi o primeiro algoritmo do tipo modelo de controle preditivo (Model Predictive Control) e é amplamente utilizado em processos industriais. Neste tipo de controlador as ações de controle são calculadas buscando minimizar uma função custo, o erro qua- 
drático entre a referência e as saídas futuras da planta. Estas saídas futuras são estimadas por um modelo linear do processo, geralmente obtido por identificação do sistema. A ação de controle do DMC é caracteriza por $\Delta u=\left(G^{\prime} G+\lambda\right)^{-1} G^{\prime}\left(y_{r}-\right.$ $f)$, onde $G$ é a matriz dinâmica da resposta ao degrau do sistema, $y_{r}$ é a referência para rastreamento de set-point e $f$ a resposta livre que depende de ações de controle passadas. Como parâmetros o DMC utiliza o horizonte de modelo $N$, o horizonte de predição $N_{y}$, o horizonte de controle $N_{u}$ e o fator de supressão e movimento $\lambda$.

Em plantas não-lineares, os erros entre o processo e o modelo linear degeneram a eficiência do controlador, criando a necessidade de modelos não-lineares. O modelo Hammerstein (Narendra and Gallman, 1966) separa a dinâmica da planta em duas partes, a primeira é o ganho não-linear estático e a segunda a dinâmica linear. No NLHDMC (Non Linear Hammerstein - Dynamic Matrix Control) o DMC é utilizado para controlar a parte linear do modelo e uma busca iterativa calcula as raízes do polinômio que representa a não-linearidade estática para encontrar o sinal de controle. As raízes complexas são descartadas e seleciona-se $u(t)$ que minimize $|u(t)-u(t-1)|$.

\section{Sintonia de parâmetros pelo modelo FOPDT}

Considerando um processo o qual possa ser adequadamente aproximado por um modelo FOPDT:

$$
\frac{Y(S)}{U(S)}=\frac{K_{p} e^{-\theta_{p} s}}{\tau_{p} s+1},
$$

discretizando o modelo acima pelo mapeamento pólo-zero $z=e^{s T_{s}}$, sendo $T_{s}$ o intervalo de amostragem, obtém-se:

$$
\frac{Y\left(z^{-1}\right)}{U\left(z^{-1}\right)}=\frac{b_{0} z^{-d}}{1+a_{1} z^{-1}},
$$

onde o atraso discreto $d=\operatorname{round}\left(\theta_{p} / T_{s}\right)+1, b_{0}=$ $K_{p}\left(1-e^{-T_{s} / \tau_{p}}\right)$ e $a_{1}=-\exp \left(-T_{s} / \tau_{p}\right)$.

Consideremos então a escolha de parâmetros $N=N_{y}=N_{u}=1$ para uma planta com atraso discreto unitário, isto é $d=1$. Esta escolha de parâmetros é perigosa na prática já que $N=1$ não descreve adequadamente o comportamento da resposta ao degrau e mal-representa a resposta livre $f, N y=N u$ pode resultar num sistema de malhafechada instável na presença de zeros de fase nãomínima. Posteriormente será mostrado que: i) o atraso não afeta a sintonia do parâmetro $\lambda$ em certas considerações parametrais; ii) os parâmetros $N, N_{y}, N_{u}=1$ tem propósito puramente de análise inicial do comportamento do DMC, afinal há alternativas melhores.

Com estes parâmetros o DMC ignora as ações passadas de controle na resposta livre, utiliza a predição de uma amostra de tempo a frente e busca otimizar apenas uma futura ação de controle. Esta escolha é fortuita para a análise em questão pois reduz o DMC ao mínimo possível permitindo uma análise algébrica simples, resultando em $G=G^{\prime}=b_{0}$ e $\Delta G=\varnothing$. A resposta livre $f=\Delta G \Delta u+y$ passa a ser $f=y$ e a ação de controle $\Delta u=\left(G^{\prime} G+\lambda\right)^{-1} G^{\prime}\left(y_{r}-f\right)$.

Manipulando a lei de controle e utilizando a estrutura RST (Sumar et al., 2009) dada por $R u=T y r-S y$ obtém-se $R=\left(G^{\prime} G+\lambda\right) \Delta, T=G^{\prime}$ e $S=G^{\prime}$. A função de transferência do sistema em malha fechada é dada por $Y\left(z^{-1}\right) / Y_{r}\left(z^{-1}\right)=$ $T B /(R A+B S)$, onde $B$ e $A$ são respectivamente numerador e denominador do modelo da planta, finalmente chega-se num sistema de segunda ordem que descreve o comportamento em malha fechada:

$$
\frac{b_{0}^{2} z^{-1}}{b_{0}^{2}+\lambda+\left(a_{1} b_{0}^{2}-\lambda+a_{1} \lambda\right) z^{-1}-\left(a_{1} b_{0}^{2}+a_{1} \lambda\right) z^{-2}} .
$$

Com esta expressão é possível sintonizar o parâmetro $\lambda$ para encontrar pólos que atendam especificações de sobre-sinal e tempo de acomodação.

\subsection{Especificação de resposta}

A especificação de resposta para a sintonia pode ser elaborada pelos métodos clássicos, como referência ver Franklin et al. (2002), utilizando $\zeta$, o coeficiente de amortecimento, e $w_{n}$, a frequência natural não amortecida do sistema. Para a especificação de sobre-sinal $M_{p}$ :

$$
\zeta=\frac{\sqrt{\log M_{p}^{2}}}{\pi^{2}+\log M_{p}^{2}},
$$

e para a especificação de tempo de acomodação de $t_{s 1 \%}$ :

$$
w_{n}=\frac{4,6}{\zeta t_{s 1 \%}} .
$$

Para encontrar os pólos alvos $z_{t}$ utiliza-se o modelo clássico de segunda ordem. Isto é, para um sistema contínuo de segunda ordem os pólos são dados por $s^{2}+\zeta w_{n} s+w_{n}^{2}=0, \operatorname{logo} s_{t 1,2}=$ $-\zeta w_{n} \pm j \sqrt{1-\zeta^{2}} w n$. Utilizando o mapeamento pólo-zero obtém-se os pólos discretos:

$$
z_{t 1,2}=e^{-\zeta w_{n} T_{s}} e^{ \pm j \sqrt{1-\zeta^{2}} w_{n} T_{s}} .
$$

Em seguida é necessário encontrar o valor de $\lambda$ que coloca o lugar das raízes o mais próximo possível de $z_{t}$.

\subsection{Sintonia do fator de supressão de movimento}

Sendo $z_{c l}$ os pólos do sistema em malha fechada obtidos pela equação 3 e $z_{t}$ os pólos desejados obtidos pela equação 6 , é necessário encontrar o va- 
lor de $\lambda$ que minimiza a distância cartesiana entre estas raízes:

$$
\min _{\lambda} \sqrt{\left(z_{t 1}-z_{c l 1}\right)^{2}+\left(z_{t 2}-z_{c l 2}\right)^{2}}
$$

resolve-se assim o problema de lugar das raízes pela variável $\lambda$, obtendo o valor ótimo $\lambda^{*}$.

Qualquer método de otimização pode ser empregado para otimizar este problema não-linear.

\subsection{Sintonia automática do fator de supressão de movimento}

Para sintonia automática (autotuning) utilizada neste trabalho, e sugerida, foi empregado um método alternativo ao anterior.

Foi utilizado um algoritmo de otimização no lugar das raízes da equação 3, neste caso um método quasi-Newton Broyden-Fletcher-GoldfarbShanno (BFGS) (Nocedal and Wright, 2006), com o objetivo de raízes $z_{c l}$ com parte imaginária nula e minimização do pólo. Resultando assim num valor ótimo $\lambda^{*}$ :

$$
\min _{\lambda} 100 \sum\left|\operatorname{imag}\left(z_{c l}\right)\right|+\sum\left|\operatorname{real}\left(z_{c l}\right)\right| .
$$

Desta maneira não é necessária a especificação manual do tempo de resposta sugerido anteriormente, já que neste caso automatiza-se uma troca entre o menor tempo de resposta e menor sobre-sinal.

\section{Estudo de Caso}

Para avaliar o método apresentado foi utilizado um sistema não-linear de válvula de controle encontrado na literatura (Wigren, 1990; Al-Duwaish and Naeem, 2001). O sistema é depictado na figura 1, adaptada de Cable (2005).

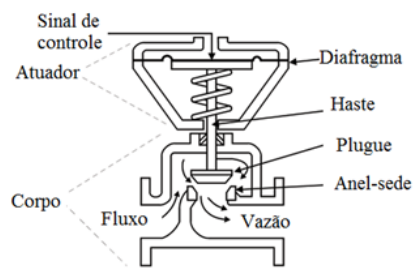

Figura 1: Diagrama do sistema válvula de controle.

Este processo é descrito pelo modelo Wiener:

$$
\begin{aligned}
& x(t)=\frac{0,616 z^{-1}+0,05343 z^{-2}}{1-1,5714 z^{-1}+0,6873 z^{-2}} u(t), \\
& y(t)=\frac{x(t)}{\sqrt{0,1+0,9 x^{2}(t)}} .
\end{aligned}
$$

O processo tem como limitação a entrada de controle $u(k)$ no domínio $[00,4]$ e tempo de amostragem $T_{s}=0,1$. A curva estática é apresentada na figura 2 onde é possível observar um claro comportamento não-linear na saída do processo.

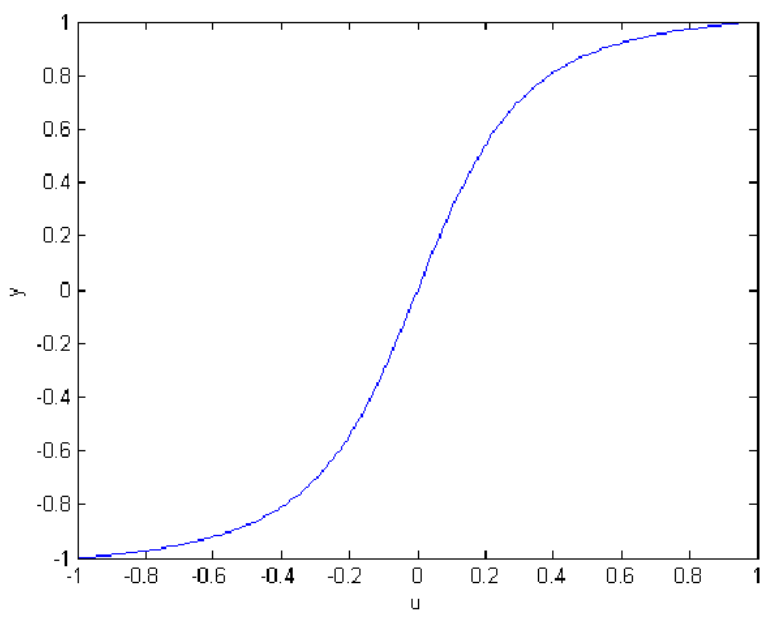

Figura 2: Característica de entrada-saída do sistema válvula de controle.

\subsection{Identificação do modelo}

Para controlar o processo com o DMC foi necessário identificar um modelo Hammerstein a partir do modelo Wiener. Para isto foi considerada uma função de ganho estático de ordem $m=3$, de grau ímpar para garantir uma raíz real, e uma função linear de ordem $n_{b}=1$ e $n_{a}=2$, isto é:

$$
\begin{aligned}
x(t) & =\gamma_{1} u(t)+\gamma_{2} u(t)^{2}+\gamma_{3} u(t)^{3}, \\
\frac{y\left(z^{-1}\right)}{u\left(z^{-1}\right)} & =\frac{b_{0}+b_{1} z^{-1}}{1+a_{1} z^{-1}+a_{2} z^{-2}} .
\end{aligned}
$$

Para identificar o modelo de Hammerstein foi elaborado o seguinte problema de otimização:

$$
\min _{b_{0,1}, a_{1,2}, \gamma_{1,2,3}} \sqrt{\frac{1}{n} \sum_{t=1}^{n}(y(t)-\hat{y(t)})^{2}},
$$

onde $n$ é o número de amostras. Novamente foi utilizado um método quasi-Newton BFGS para a tarefa de otimização, resultando em $b_{0,1}=$ [-1,593896845712190 0,704732341842690], $a_{1,2}=[2,797526045953196-0,862005679935081]$ e $\gamma_{1,2,3}=[0,194536814826656$ 0,200460554117093 0,007938804763741], com $R M S E=0,006$ (Root Mean Squared Error). O resultado final da identificação é exibido na figura 3.

\subsection{Sintonia e controle DMC}

Para identificação do modelo FOPDT foi utilizado o método proposto em Smith (1972) resultando 


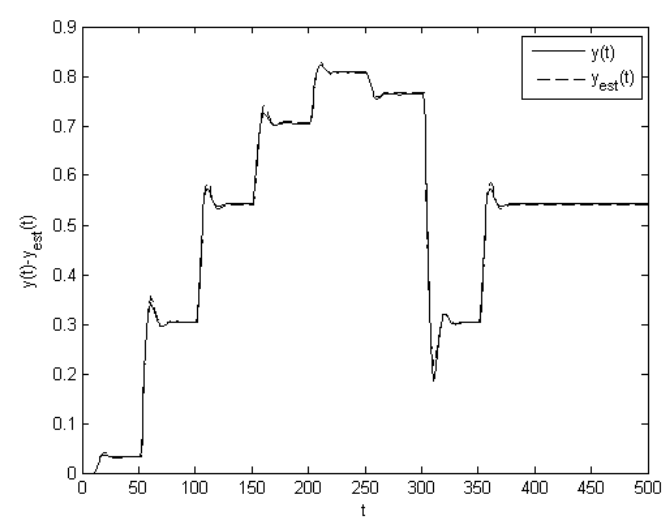

Figura 3: Saída do sistema válvula de controle e sistema equivalente Hammerstein identificado.

em $K_{p}=17,4658, \tau_{p}=0,2478$ e $\theta_{p}=0,1767$. Em seguida são considerados 3 casos de sintonia:

\section{Sintonia 1}

Neste primeiro caso é utilizado para comparação um método de sintonia bem conhecido da literatura proposto por Shridhar and Cooper (1997).

$$
\begin{aligned}
N & =\left\lceil 5 \frac{\tau}{T_{s}}+\left\lceil\theta_{p} / T_{s}+1\right\rceil\right] \\
N_{y} & =N \\
1 & \leq N_{u} \geq 6 \\
\lambda & =\frac{N_{u} K_{p}^{2}}{500}\left(3,5 \frac{\tau}{T_{s}}+2-\frac{N_{u}-1}{2}\right) .
\end{aligned}
$$

\section{Sintonia 2}

Neste segundo caso é utilizada a sintonia de análise proposta anteriormente, isto é:

$$
\begin{aligned}
N & =1, \\
N_{y} & =1, \\
N_{u} & =1, \\
\lambda & =\lambda^{*} .
\end{aligned}
$$

Embora interessante para análise, esta sintonia é pobre do ponto prático pois $N=1$ ignora quase toda a resposta ao degrau, assim como qualquer elemento de atraso. Outro problema é que $N_{y}=N_{u}=1$ resulta em pólos instáveis de malha-fechada na presença de plantas com zeros de fase não-mínima.

A vantagem desta sintonia é que nestas condições parametrais o ajuste do fator de supressão de movimento tende a ser extremamente conservativo já que o horizonte de controle é o menor possível e é possível a sintonia buscando uma resposta específica.

\section{Sintonia 3}

Para melhorar a sintonia anterior é necessário considerar o tempo de resposta assim como o atraso do sistema. Com estes conhecimentos da dinâmica é possível o controle pelo DMC de processos de fase não-mínima e altos valores de tempo morto. Com a incorporação do tempo morto à sintonia de $N$ e $N_{y}$ o coeficiente de supressão de movimento $\lambda$ se torna independente do atraso (Shridhar and Cooper, 1997), justificando a desconsideração do atraso na sintonia de $\lambda$.

Ao considerar o tempo de acomodação, atraso do sistema e futuras ações de controle, a nova sintonia coincide então com um híbrido da proposta por Shridhar and Cooper (1997) com a proposta neste trabalho:

$$
\begin{aligned}
N & =\left\lceil 5 \frac{\tau}{T_{s}}+\left\lceil\theta_{p} / T_{s}+1\right\rceil\right\rceil, \\
N_{y} & =N \\
1 & \leq N_{u} \geq 6, \\
\lambda & =\lambda^{*} .
\end{aligned}
$$

Para a sintonia buscou-se sobre-sinal nulo e minimização do tempo de acomodação usando novamente o método quasi-Newton, resultando em $\lambda^{*}=850,1$. O lugar das raízes para o segundo e terceiro caso em função de $\lambda$, é apresentado na figura 4 , indicando o valor obtido e a melhor troca entre tempo de acomodação e sobre-sinal. Os parâmetros para todos os casos de sintonia estão dispostos na tabela 1 .

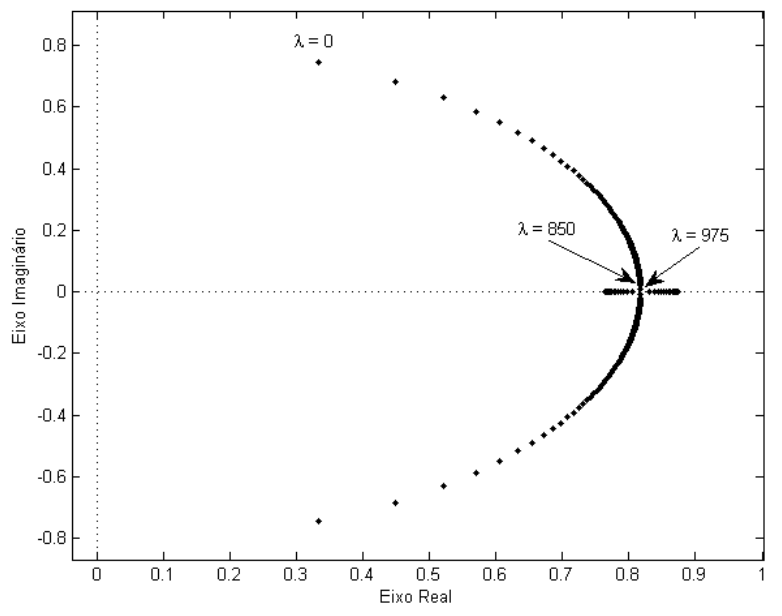

Figura 4: Lugar das raízes para o sistema válvula de controle em função de $\lambda$. 


\begin{tabular}{c|c|c|c|c}
\hline Sintonia & $N$ & $N_{y}$ & $N_{u}$ & $\lambda$ \\
\hline 1 (Shridhar e Cooper) & 16 & 16 & 5 & 26,46 \\
2 (puro) & 1 & 1 & 1 & 850,1 \\
3 (híbrido) & 16 & 16 & 5 & 850,1
\end{tabular}

Tabela 1: Parâmetros dos casos de sintonia

\section{Resultados}

O sistema não-linear foi simulado buscando controlar pontos de referência ao longo de toda a faixa não-linear de operação. Foram utilizadas 9 variações de set-point a cada 5 segundos para um total de 50 segundos de simulação. Para $T_{s}=0,1$ isso equivale a 500 amostras. Cada método de sintonia é apresentado numa simulação separada exibindo resposta do sistema e da ação de controle nas figuras 5,6 e 7 .
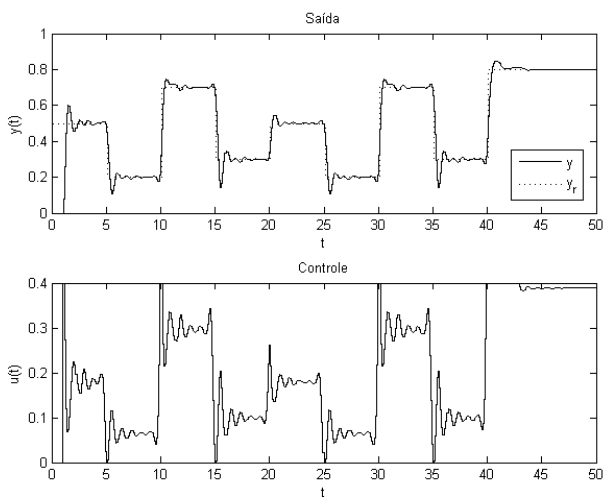

Figura 5: Resposta do sistema para a sintonia de Shridhar e Cooper (1997).
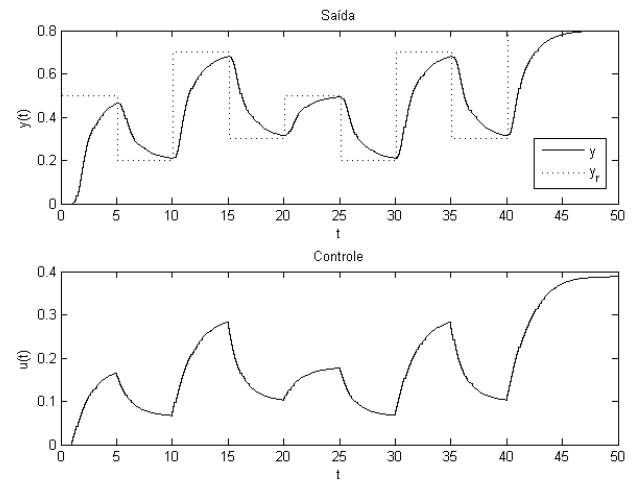

Figura 6: Resposta do sistema para a sintonia proposta pura.

Para a primeira sintonia observa-se que o método de Shridhar and Cooper (1997) apresenta uma resposta extremamente agressiva assim como uma ação de controle acentuadamente oscilatória.

Em contra-partida para o método de sintonia pura o sistema apresenta uma resposta extremamente sobre-amortecida e um tempo de acomoda-
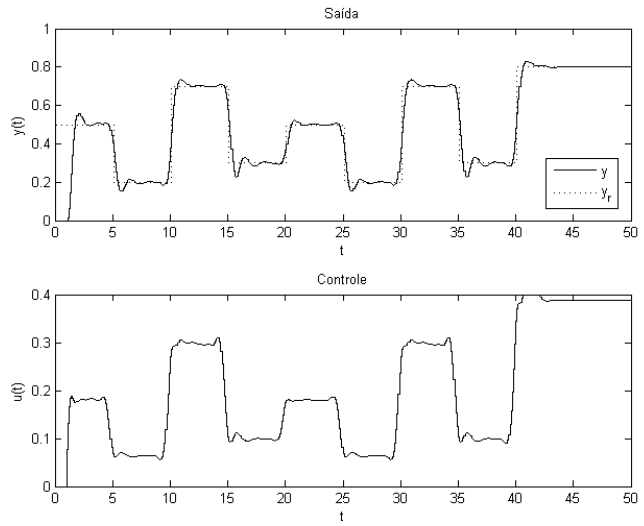

Figura 7: Resposta do sistema para a sintonia proposta híbrida.

ção alto, acima de 5 segundos. Conforme esperado o sistema apresenta-se demasiadamente conservativo na ausência de conhecimento das respostas futuras e da consideração de apenas uma ação de controle.

Já para a terceira sintonia, o método híbrido, o sistema apresenta uma resposta muito mais adequada tanto quanto ao tempo de acomodação assim como uma ação de controle suave. Lembrando que esta resposta foi obtida por uma sintonia completamente automática (autotuning).

Sabe-se das considerações anteriores que a segunda sintonia tem propósitos apenas de estudo e não práticos. Pelos resultados observados a sintonia híbrida proposta neste trabalho apresenta melhor desempenho em relação a sintonia de Shridhar and Cooper (1997).

\section{Conclusões}

Neste trabalho foi apresentado um método de sintonia para o DMC com análise da resposta do sistema sob determinados parâmetros. Através desta análise inicial chegou-se numa sintonia conservativa para o fator de supressão de movimento da ação de controle. Entretanto, esta análise inicial teve como propósito apenas a sintonia do fator de supressão de movimento já que devido as pobres considerações da dinâmica do processo a sintonia inicial se apresenta como demasiadamente conservativa para o DMC. Para tornar a ação de controle mais adequada foi necessário expandir a sintonia inicial para que o DMC contemple o tempo de acomodação e atraso do sistema.

Ao considerar o tempo de acomodação, atraso do sistema e futuras ações de controle, a nova sintonia passou a coincidor então com um híbrido da proposta inicial com uma sintonia popular na literatura e proposta por Shridhar and Cooper (1997).

Foram propostos também um método para 
sintonia manual com especificação de resposta e um método de sintonia automática (autotuning). Neste trabalho foram contemplados resultados apenas da sintonia automática.

Um sistema não-linear de válvula de controle descrito por um processo de Wiener foi utilizado para as simulações. O sistema foi transformado em um modelo de Hammerstein para ser utilizado com o DMC. Os resultados obtidos demonstram que a sintonia automática com abordagem híbrida proposta neste trabalho resulta em bons tempos de resposta assim como uma ação de controle suave se comparada com a de Shridhar and Cooper (1997).

Trabalhos futuros irão se concentrar em: i) alterar a função objetivo da sintonia manual para um somatório de quadrados do tipo MSE (Mean Squared Error) e utilizar otimização por mínimos quadrados não lineares; ii) utilizar um método de otimização como busca do gradiente durante o processo de controle para refinar o valor de $\lambda^{*}$ na presença de não-linearidades dinâmicas.

\section{Agradecimentos}

O primeiro autor agradece a UFSC pela concessão de uma bolsa de doutorado vinculada ao CNPq (processo: 140592/2012-1).

\section{Referências}

Al-Duwaish, H. and Naeem, W. (2001). Nonlinear model predictive control of hammerstein and wiener models using genetic algorithms, Control Applications, 2001. (CCA '01). Proceedings of the 2001 IEEE International Conference on, pp. 465-469.

Cable, M. (2005). Calibration: A Technician's Guide, ISA Press, Research Triangle Park, USA.

C.R., C. and P.S., R. (2001). Dynamic matrix control - a computer algorithm, Proceedings of the Joint Automatic Control Conference, San Francisco, CA, USA, pp. no. WP5-B.

Cutler, C. R., Prett, D. M. and Ramaker, B. L. (1982). Dynamic matrix control method, United States patent US 4349869, issued 1982-09-14.

Franklin, G. F., Powell, J. D. and Emami-Naeini, A. (2002). Feedback Control of Dynamic Systems, 4th edn, Prentice Hall.

Kokate, R. D., Waghmare, L. and Deshmukh, S. D. (2010). Review of tuning methods of dmc and performance evaluation with pid algorithms on a fopdt model, Advances in Recent Technologies in Communication and
Computing (ARTCom), 2010 International Conference on, pp. 71-75.

Narendra, K. S. and Gallman, P. G. (1966). An iterative method for the identification of nonlinear systems using a hammerstein model, IEEE Transactions on Automatic Control 11(1): 546-550. DOI: 10.1109/TAC.1966.1098387

Nocedal, J. and Wright, S. J. (2006). Numerical Optimization, 2nd edn, Springer, New York.

Qin, S. J. and Badgwell, T. A. (2003). A survey of industrial model predictive control technology, Control Engineering Practice 11(1): 733-764. DOI: 10.1016/S0967-0661(02)00186-7

Shridhar, R. and Cooper, D. J. (1997). A tuning strategy for unconstrained siso model predictive control, Industrial \& Engineering Che-mistry Research 36(1): 729-746. DOI: 10.1021/ie9604280

Smith, C. (1972). Digital computer process control, International Textbook Company, Scranton, Pennsylvania.

Sumar, R. R., Coelho, A. A. R. and dos Santos Coelho, L. (2009). Use of an artificial immune network optimization approach to tune the parameters of a discrete variable structure controller, Expert Systems with Applications 36(3, Part 1): 5009-5015. DOI: 10.1016/j.eswa.2008.06.011

Wigren, T. (1990). Recursive identification based on the nonlinear Wiener model, $\mathrm{PhD}$ thesis, Uppsala University, Disciplinary Domain of Science and Technology. 